\title{
Cultural Life: Variation, Selection, and Homeostasis
}

\author{
Author: Dan C. Baciu (ORCID: 0000-0002-0043-5616. University of California, Santa
}

Barbara, Department of English, Department of History of Art and Architecture)

\begin{abstract}
:
Cultural life renews itself constantly, uniting people, and diversifying thought. In this process, new thought emerges, and new varieties depart further and further away from pre-existent types. To study this evolution, the present article defines large-scale variation and selection and models them as variation-selection processes. On this basis, empirical testing reveals that cultural life accommodates many parallel variationselection processes. In support of cultural diversification, homeostatic regulation balances their interplay. Real-world effects are measurable in pervasive cultural phenomena: large-scale polarization, renegotiation of shared values, returning fashions, formative periods, cycles of growth and reform, common ethos, and beyond. To test predictions, data from hundreds of thousands of books, news, television, and social media have been collected, decoded, and enriched with geographical and historical information. The discovery of variation-selection processes and homeostatic regulation in cultural life only complements a larger set of discoveries that have united physics and biology into modern life science.
\end{abstract}

\section{Introduction}

History repeats itself: Fashions return; polarization persists and prevails; and periods of growth and reform alternate, spiraling in endless cycles along the time axis. At the same time, new public awareness forms and rises, and new collective dreams replace old ones.

In the last few decades, some of these recurrent patterns have become quantifiable across digital sociology and humanities as well as computational psychology and linguistics (Piantadosi, 2014; Landauer and Dumais, 1997). Evidence converging from these disciplines confirms for example that polarization has the ability to self-perpetuate, and that culture at large is distributed unevenly. Distributional laws describe divides between famous people and those unknown as well as between top ten ranking items and the long tail of everything else.

In parallel to such empirical findings, the general public has become acquainted with quantitative evaluations of cultural change (Michel et al., 2011). Many online platforms allow everyone to interactively trace the usage of words, terms, and phrases over time. Suddenly, data journalists can easily awaken fascination in memes; and terms such as fashions, trendsetters, and pyramids of perception and knowledge have entered common usage and help people speak about culture at large.

Additional qualitative observations come from historians. For example, historians are often forced to attest that a common ethos can evolve to unite entire bodies of knowledge. However, such unison is often reached only after extended periods of formation (Baciu, 2018, 2019, 2020, Abramova and Baciu, 2020a, b, Baciu and Abramova 2020). Paradoxically, common ethos works hand in hand with diversification. 
In response to these empirical discoveries as well as to the new public-facing technologies, this present article develops and tests a new theory of cultural life based on variation-selection processes and homeostatic regulation. The latter is divided into three sub-processes, namely, habituation, discrimination, and sensitization. This level of detail makes it possible to explain, interconnect, and predict the phenomena mentioned above and beyond. The theory has been first proposed in previous work (Baciu 2017, $2018,2019,2020 a, b)$, but the present article discusses it specifically for humanities scholars and social science researchers.

Variation-selection processes and homeostasis are well known in the life sciences, but they were applied to the small scales of individuals and not the large scale of cultural life (Schulkin, 2003). The new discovery not only reconfirms that these processes are ubiquitous, but it also leads to new conclusions that may be valid and valuable across multiple domains of knowledge.

In particular, diversification appears to be a main driving force that controls both variation-selection processes and homeostasis. The present article develops the concept of diversity selection for processes that are fine-tuned to reap the benefits of diversification. Such processes may constitute a sixth type of cooperation next to the five types previously classified (Nowak, 2006b).

With respect to cultural life, the new theory has many potential applications. First, it may help people better understand culture and make more informed decisions for their lives. Culture is vast and may seem difficult to see through. If you are a player on a soccer field, you best understand your own role, but it is nevertheless beneficial to understand the whole game. Of course, life is more complex than a soccer game, but if the general public is able to understand something as complex as the human brain, there are few limitations to fear.

For example, a better understanding of formative periods may allow people to speed up the formation of public opinion where action is needed. Environmental awareness could rise sooner, not lagging behind pollution, as it often does (Abramova and Baciu, 2020a, b).

Similarly, a better understanding of the forces that shape the evolution of a common ethos may help people reach consensus. And even further, a better understanding of polarization may resolve conflict; and a better understanding of cultural diversification may inspire us to efficiently advocate for it.

Admittedly, cultural life is still complex and puzzling. The present article therefore proceeds step by step, beginning with simple observations, developing simple models, and adding additional complexity over the course of each section. The added complexity is tested at every stage separately.

The new theory is not a standalone. It covers and has connections to multiple fields of study ranging from evolutionary dynamics to physical chemistry, physiology, immunology, virus dynamics, history, mathematics, evolutionary psychology, economics, as well as digital humanities and sociology.

These topics are not typically studied in conjunction. It is therefore recommended that the reader go through the text twice, the first time to familiarize him or herself with the matter, and the second time to comprehend it. The sections that are set aside as tests explain the mathematics and testing procedure in plain language. As such, the tests are relevant mostly to the advanced reader. 


\section{Overview}

Section 1 introduces and defines reproduction and variation.

Section 2 makes the logical connection between reproduction, variation, and selection. Their interplay is formulated as variation-selection processes.

Section 3 predicts distributions and dynamics using variation-selection processes.

Test A confirms the predictions for one isolated variation-selection process.

Test B confirms the predictions for multiple parallel variation-selection processes.

Context A places theory and test results into the broader scientific context.

Until section 3, the article deals with variation-selection processes as applied to dissemination by authors and publishers. From section 4, the theory is expanded to homeostatic regulation as applied to reception in large audiences.

Section 4 draws attention to the fact that variation-selection processes are often fine-tuned to maximize diversification. Such diversification can yield many benefits. As a result, it is postulated that additional processes can evolve to support the evolution of diversification. The concept of diversity selection is developed; and it is identified as a distinct type of cooperation.

Section 5 suggests that homeostatic regulation may allow for additional diversification by balancing multiple variation-selection processes. To empirically operationalize this hypothesis, homeostasis is further split into habituation, discrimination, and sensitization.

Section 6 predicts that interplay between media activity and habituation can lead to returning fashions. Test $\mathbf{C}$ confirms the predictions.

Section 7 predicts that formative periods are periods of diversification. When a certain diversity threshold is reached, diversification turns into growth.

Test $\mathbf{D}$ confirms the predictions.

Section 7 predicts that interplay between variation-selection processes and homeostasis leads to cycles of growth and reform. The cycles are named "diversification cycles."

Test $\mathbf{E}$ confirms the predictions.

Section 8 makes additional predictions about the formation of common ethos. A qualitative test is referenced from elsewhere.

Sections 9 and 10 place the results into the broader scientific context. 


\section{Varieties permeate all realms of thought.}

Cultural practices require repetition, often without change. The same music is played and replayed, or else it fades away. The same theatrical compositions are retaken, or else they die. The same artworks travel around the world to be exhibited and discussed. The same texts are reprinted and reread. The same scientific experiments are replicated across many universities. Finally, monuments are erected and preserved to cement wellestablished cultural values, and to keep them alive by constantly reminding people of past deeds.

However, change occurs along the way. New music is improvised and composed. New plays are written. New schools of thought form, branch, and split. Sciences and humanities diversify. In this process, research and critical thinking make use of new and newer sets of criteria to test many similar theses and hypotheses.

Creativity, too, leads to the formation and spread of multitudes of varieties. Designers think in variants and alternatives; authors write and rewrite; games exist in multiple versions; and lifestyles change. Furthermore, scientific work on memory has shown that memories, too, are rewritten when they are recalled, which may gradually transform them (Besnard and Laroche, 2012; Shaw, 2017).

Taken together, one can say that cultural life can reassure as well as surprise. Repetition is accompanied by variation. Let us call this observation variation principle. The underlying insight is that thought diversifies while it is being reproduced. As a logical result, new varieties emerge coinciding in space and time with pre-existent types.

In communication theory, Claude Shannon developed a framework that reflects both repetition and variation. He defined communication as exact or approximate reproduction of a message at a new point (Shannon, 1948). Repetition is exact reproduction. Variation is approximate reproduction.

\section{Variation on a large scale leads to variation-selection processes.}

Shannon's original theory of exact or approximate reproduction of messages was developed as part of a larger effort to free telecommunication from noise. As a consequence of this historical context, approximate reproduction was mostly studied with respect to noise that interferes during message transmission.

The process is easy to illustrate: A message is reproduced in presence of noise. The initial recording may be of impeccable clarity. However, over time, the copy, and the copy of the copy, etc. can only become increasingly noisy. After repeated reproduction, the initial message eventually fades away behind noise. Information entropy is accumulated (Shannon, 1949).

Even if Shannon's theory is expanded to include any type of variation, the same result is obtained again. Take the example of a complex technology. Any single change is more likely to devalue such technology. On average, the accumulation of single changes is detrimental (Henrich, 2017).

The theory of large-scale dissemination proposed in this article goes one logical step further and predicts the opposite result. Multiple messages are exactly and approximately reproduced in parallel. This small change in the initial definition means that pre-existent and new thought can come to stand next to each other in space and time. Evolution can compare variants and search in multiple directions. Each variant constitutes a separate search direction, or, in other words, each variant is an additional 
option to choose from. As before, the majority of newly produced copies will be disadvantageous. However, one advantageous option is enough for the process to restart from this new and more promising point. This type of evolution is known as variationselection process. Brainstorming is a similar process in that multiple alternatives are created and selected from in parallel. (Another such process is Fritz Zwicky's morphological approach for problem solving. This approach envisions breaking research questions into sub-questions and evaluating multiple combinations in parallel.)

Variation-selection processes are well known in physical chemistry. The quasispecies equation is the classical framework to study these processes in molecular genetics (Bertels, Gockhale, and Traulsen, 2017; Domingo, Schuster, and Oldstone, 2016; Nowak, 1992; Eigen, 1971). In the quasispecies equation, information is exactly as well as approximately reproduced in parallel. Quasi-species means approximately reproduced species, which is equivalent to the definition of variation-selection process already developed above.

Given that the quasispecies equation already exists, let us use the terminology and mathematics already developed in that context. This means that we must make a couple of connections between physical chemistry and cultural life.

The quasispecies equation was first developed for chemical species. However, the term species has much broader meaning. The term's linguistic root goes back to the Latin word for seeing and discerning, and is also found in the more common words spectator and special. In the broadest sense, each species is a category of items that are special, i.e. clearly distinguishable from other items. Already in Antiquity, Aristotle categorized objects and ideas by the characteristics that made them special. Those special characteristics were eventually called specific differences.

Over the course of the millennia, Aristotle's method was applied to the study of nature as well as culture. Eventually, the term species came to denominate the standard unit of classification in the physical and natural sciences.

In the humanities, the term species is uncommon. Instead, ideas are taken to be the basic unit into which thought is classified. Ideas are clearly distinguishable categories of thought. Similar to species, the term idea goes back to a historical word root for seeing. This time the word root is taken from ancient Greek rather than Latin.

The terms idea and species are abstractions. The same can be said about varieties and variants. More broadly, experimenters are free to define under which circumstances they put two items into different categories, or into one and the same category. In chemistry, species are categories of identical molecules, for example all $\mathrm{CO}_{2}$ molecules form one chemical species. In molecular genetics, species are identical chains of nucleic acids.

In contrast to regular species, quasispecies allow for approximate reproduction. In molecular genetics terminology, this means that quasispecies allow for mutations: point mutations, deletions, insertions, and recombination (Nowak, 2006a, 35; point mutations exchange one single nucleic acid with a different one; deletions remove nucleic acids; insertions insert new ones; recombination recombines multiple chains).

In humanities terminology, approximate reproduction means departure. New ideas depart from pre-existent ones. Departure can simplify, complicate, or recombine pre-existent ideas by adding, removing, or recombining characteristic features.

Different sciences have developed different terminologies. Next to quasispecies, the word spectrum is also in use. In physics, light spectra are collections of light of similar wavelength. In psychiatry, spectrum disorders are groups of similar conditions. 
The contribution of the quasispecies equation to modern science is not the broadening of the term species. The real merit of the equation is that it can be used to predict the effects of exact and approximate reproduction on a large scale-and this is what we are after, too.

Let us illustrate variation-selection processes in a little more detail with a simple example. During your daily life, many ideas catch your attention. Sometimes, you choose one and share it with someone else. Technically, this means that you reproduce that idea. As a result of sharing, there are suddenly two copies of it. Moreover, you may have added something new to the copy you shared, making this new copy clearly distinguishable from the previous template that initially attracted your attention. One could say that template and copy are two ideas, but to adhere to standard terminology, we will call these ideas variants. The two variants that have just been created are a quasispecies, albeit a rather small one. One important property of the quasispecies is already observable: Variation connects the two variants with each other, but they are not identical, and this can push evolution into one or the other direction. Over time, the quasispecies grows as copies depart further and further away from the original templates.

The quasispecies equation expresses variation-selection processes in mathematics, which allows us to predict distributions and dynamics. We can predict how many copies there are of each variant at any given time.

As mentioned before, the division of the world into chemical, biological, and cultural categories is a theoretical construct. Nevertheless, this categorization allows for the study of distributions that exist independent of theory. Similarly, the metric unit to measure space is arbitrary. You can use meters, miles, or some other metric. However, landmasses are distributed the way they are before the metrics are devised. At first, this sounds like another tiny logical step, but its value depends on the phenomena that it can help us understand.

\section{Explaining why power laws and clustering patterns self-perpetuate.}

The new theory of dissemination explains, in a single stroke, the two most pervasive distributional laws of human culture, namely, power laws and clustering patterns. Let us proceed by making our previous thought experiment a little more concrete. Rather than thinking abstractly of an idea that spreads and diversifies, we can choose a real-world example that will later serve for testing.

Imagine collecting everything called Chicago school. A first inspection of the data immediately reveals that there are many schools of thought that people have dubbed Chicago school over the course of time. Some schools are found in social science, others in architecture, art, and entertainment. There are schools named after a person, a method, or an entire research direction. To distinguish between these schools, people coined recurrent expressions that are partially ambiguous and partially redundant but nevertheless allow us to categorize the Chicago schools into clearly distinguishable variants. What would we expect to observe if we counted how many times each of these variants was mentioned in public media?

With a little life experience, one would expect that some schools are frequent, while others account for the long tail of less prolific variants and alternatives. Such distribution is typically compared to the pyramid of income: A few winners take most. Even fictional worlds invented on the spot obey this law (Piantadosi, 2014). 
In addition, one would also expect to see schools cluster into groups. Polarization would occur between the groups. To illustrate this type of distribution, past researchers and philosophers have coined the term topics (Landauer and Dumais, 1997). The same word root is also found in topography. Next to the long-tailed distribution, the clustering patterns may be the second most pervasive empirical law of human culture.

The quasispecies equation can be used to predict dynamics and distributions, and the predictions are in accord with empirical data (Fig.1). Tests A and B go through the mathematics. Once again, our proposition is that thought emerges through variationselection processes. In turn, thought is expressed in language; that is, words, terms, and phrases, the distributions of which come to be representable as power laws and clustering patterns. Now these distributions are also predictable through the quasispecies equation.

The mathematics is not everyone's darling, but we can also expand the figurative example of landmasses. In this example, the material rolling to the sides symbolizes variation. This process determines the slopes of the landforms. Once again, the unit of space chosen to measure the topography is somewhat arbitrary. However, the slopes of the landscapes are not. If no material rolls to the sides at all, the hills are steep, if not overhanging cliffs. In contrast, if the same material is loose sand, the hills are flat dunes.

\section{Test A: One quasispecies.}

Variation-selection processes can be mathematically formulated, which makes it possible to predict distributions and dynamics. The simplest case is one isolated variation-selection process. A little more terminology is helpful. As before, copies are being reproduced. Variants are distinct categories of copies. Exact reproduction means that a new copy has been produced, and that the new copy and the original template both belong to the same variant. In contrast, approximate reproduction means that copy and original template belong to two different variants.

Approximate reproduction is also called transition. The term transition is chosen to reflect that copies transition from one variant to another. In humanities terminology, transition is referred to as departure from previous knowledge.

Accordingly there are two main types of reproductive rates, exact and approximate. Approximate reproductive rates are also called transition rates. The transition between any two arbitrarily chosen variants may occur at its own average rate. This means that in a system of $n$ variants, there are $n$ exact reproductive rates and $n^{2}-n$ transition rates. Together, these make a total of $n^{2}$ values.

In principle, there are many ways in which such information can be stored. One could choose networks or graphs, (or one could write down the information in full sentences). The choice of the data structure is arbitrary. Let us therefore adhere to the choice already made by the quasispecies equation.

In the context of the quasispecies equation, the information is stored as a square matrix. For $n$ variants, the matrix has $n$ rows and $n$ columns. This makes a total of $n^{2}$ fields. The diagonal contains the exact reproduction rates. All other fields are pairwise transition rates. (For example the transition rate from variant 1 to variant 2 is stored in the first row second column. As before, the variants are somewhat arbitrary, but the transition rate is not.) This matrix containing all reproduction rates, exact and approximate. Nevertheless, because there are so many transition rates, the matrix is also called transition matrix. 
Rather than attempting to empirically measure the total $n^{2}$ quantities that go into the transition matrix, the research concept is to simplify the model to such extent that only little measurement is necessary. For the study of one isolated quasispecies, the model can be transformed such that all that is need is a taxonomy of the existing variants. And even this taxonomy can be further simplified.

Fist, there is a wild type variant that reproduces fastest. Second, there is an entire group of variants that immediately surround the wild type. Third, there is a group consisting of all variants that surround this previous group of variants. Finally, there is a group of all remaining variants (Bertels, Gockhale, and Traulsen, 2017). Figuratively, imagine a drop of water that creates four concentric ripples in a cup.

This geometry makes it easy to estimate reproduction rates. Three assumptions are sufficient to generate all values that must be estimated to run a basic experiment.

The first assumption is that the closer a variant is to the wild type, the higher its reproductive rate. This also means that the closer the entire group is to the wild type, the higher the entire group's average reproductive rate.

This simplification means that there is no need to store all $n$ variants in the matrix. Some rows can be aggregated. There only need to be rows and columns to store those four previously mentioned groups of variants. A four-by-four matrix can do the job.

The second and third assumptions are that most transition only occurs between immediate neighbors, and that transitions rates between variants that are immediate neighbors are the same.

Based on this total of three assumptions, it is possible to estimate all required transition rates. In our four-by-four matrix, only six values are greater than zero. In turn, these transition rates fall into two categories. Transition rates away from the wild type and those towards it.

The systematic of four concentric groups of variants has a property that affects transition rates: the further away from the wild type, the larger the potential size of the group. This translates into larger transition rates away from the wild type and smaller transition rates towards the wild type in the four-by-four matrix. The same logical conclusion applies in biology (Bertels, Gockhale, and Traulsen, 2017).

In sum, the estimated parameters for the model are as follows: four linearly decaying replication rates along the diagonal of a four-by-four matrix, three equal transition rates above the diagonal, three smaller transition rates below the diagonal.

To solve the equation, four additional numbers are needed. They represent the initial distribution of how many copies initially exist within each of our four groups of variants. This distribution does not affect the final steady-state distribution, which makes the choice arbitrary for our present purpose. The only thing that matters is to store these for numbers in a vector that can be multiplied with the matrix. A unit vector can do the job.

The next step is to solve the equation. The vector that represents the initial distribution is multiplied by the reproduction and transition rates stored in the transition matrix. This operation simulates the passage of time; it gives a new vector that represents the distribution after one round of reproduction. These new numbers are then multiplied again by the same reproduction and transition rates, (assuming that the reproduction rates remain the same after each round of reproduction). Each multiplication represents one round of reproduction in the population. Eventually a steady state is reached at which the distribution does not change. The system will 
always tend towards this steady-state equilibrium. Formulated as matrix operation, this type of iterative multiplication is known as power iteration. We will return to this operation later.

The results for the four-by-four matrix are shown in figure 1. Stochastic refinements were replicated from Bertels, Gockhale, and Traulsen, 2017, where they were developed towards a different purpose.

The historical data on Chicago schools was obtained from historical research presented elsewhere (Baciu, 2018, 2019). The quasispecies prediction and the empirical data on four groups of Chicago schools of sociology show the same distribution, hence confirming the prediction.

Fig.1 One quasispecies. Variation-selection processes among Chicago schools of sociology and related fields. Records from public media are structured into four groups of schools. The prevalence of each group is estimated using the quasispecies equation. The results are within the expected boundaries. Stochastic refinements from Bertels et al. 2017.

A Quasispecies transition between groups. B Quasispecies evolution over time (left). Quasispecies distributions (right). C Distributions from empirical data on Chicago schools of sociology and related fields. (Data: Chicaog school corpus, $y=1 \mathrm{~K}$ references.)

\section{Wild type 2. Variants sourrounding 1. 3. Variants surrounding 2. 4. All other variants.}
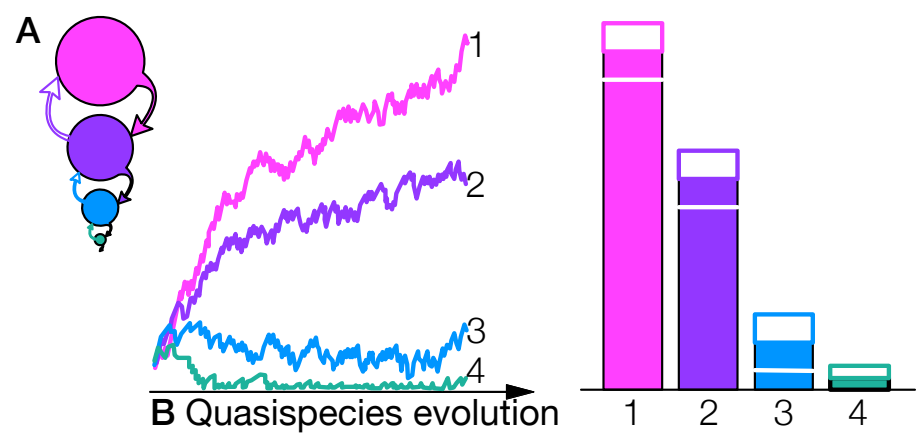

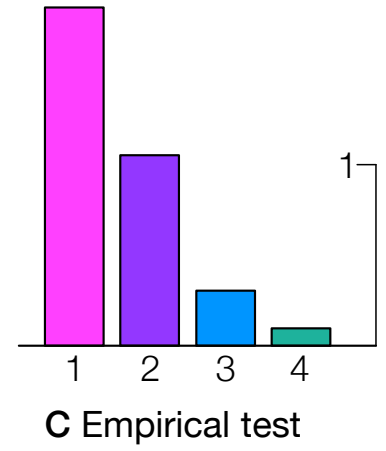

C Empirical test

\section{Test B: Multiple quasispecies.}

Mathematically, it can be demonstrated that the fastest reproducing quasispecies must eventually come to predominate. However, cases of viral quasispecies are known in which immune responses check the growth of this dominant quasispecies, chasing virus evolution away from it (Nowak, 2000, 89).

Such observations have consequences for the study of variation-selection processes in cultural life as well. In Test A, one quasispecies predominated. However, such finding could be a mere special case. The other possibility is that traces of multiple quasispecies are found in parallel. If this latter result is found, it means that variationselection processes are likely to be at work in concert with other processes.

Let us proceed to setting up the experiment. Test A has introduced the quasispecies equation and studied one, dominant quasispecies. In that context, it 
seemed easy to categorize all variant ideas into four concentric groups. However, the same simplification does not hold when multiple quasispecies are studied because each quasispecies could have its own center. The groups are no longer concentric. Figuratively, the ripples on the water interfere with each other.

The search for a different approach brings us back to one of the first observations that guide this article: new thought emerges coincident in space and time with preexistent thought. This statement can be logically deduced from the variation principle, and it also opens up multiple paths to estimating transitions.

A little more terminology is helpful. Pre-existent and new thought occurs together at separate instances in space and time. At each of these instances, pre-existent and new thought co-occurs.

Instances can be defined at different granularities. Each library, each book, each article, each sentence could be defined to represent a separate instance in space and time. Similar to defining variants, this granularity is somewhat arbitrary.

However, no matter how the granularities are chosen, one basic fact remains the same: each variant has a number of copies that belong to it, and all of these copies are distributed over a number of separate instances in space and time.

Let us call the total number of copies of any given variant at any given instance the variant frequency. Technically, all variant frequencies can be collected and represented as a rectangular matrix. The rows represent the variants, the columns the instances. Each field represents a distinct variant frequency. This matrix is can also be called a co-occurrence matrix because rows and columns record variants co-occurring at separate instances.

High variant frequencies mean that the variant has been reproduced many times. Therefore, they mean high reproductive rates. Thus, the co-occurrence matrix could be seen as a rough estimate of a transition matrix similar to the one used in the quasispecies equation. Nevertheless, the co-occurrence matrix can be further transformed to better represent transition rates.

One observation can easily be made after inspecting the data. Each variant is distributed over an entire number of separate instances. Let us refer to this number as instance frequency. (For example: if a variant occurs ten times at instance one and twenty times at instance seven, but it did not occur at any other instances, its instance frequency is 2 because it occurred at two instances.) The higher the instance frequencies of two terms are, the higher is the likelihood that the co-occurrence of these two terms does not represent transition but mere chance. Therefore, the mathematical operation needed is to divide the variant frequencies by instance frequencies.

As a concrete example, consider a document-term-matrix. The matrix consists of rows for each distinct term and columns for each of the documents. These are the equivalents of variants and instances in this concrete setting. The order of rows and columns is arbitrary. The data stored in the matrix are total counts: If the first term is found five times in the second document, it means that the first row, second column will have the value 5 .

Once again, two basic facts are evident. If there are many copies of a given word, it means that this word is reproduced fast. However, if these copies are distributed across many separate documents, this increases the probability that co-occurrence is mere chance. The operation needed is to divide the total count of a given word in a given document by the total number of documents in which this word occurs. With respect to term-document-matrices, this procedure is known as tf-idf (Spärck Jones, 1972). 
An additional step is not mathematically necessary, but it allows us to stay within the quasispecies terminology given in Test $\mathrm{A}$. There, the reproductive rates are stored in a square matrix. The rows and columns represent the $n$ variants. The diagonal represents exact reproductive rates. And all other fields are pairwise transition rates. As mentioned before, this choice is arbitrary. Technically, the square and rectangular representations of the matrix are interchangeable.

The transformation from rectangular to square matrix is a mere data processing step. The rectangular variant-instance-matrix is multiplied with an inverse instancevariant-matrix. This operation gives the required variant-variant-matrix.

Now that we know how to build the matrix, let us solve the equation. The power iteration procedure mentioned before can be generalized to what is known as eigendecomposition. If the rectangular matrix is kept, the equivalent result is achieved by applying singular value decomposition. Through this procedure, we obtain multiple independent vectors, the eigenvectors of the matrix. These vectors are the independent axes of the matrix. In our setting, each vector represents a quasispecies. Compared to Test A, now there are multiple quasispecies.

After the equation is solved, the question arises how to interpret and test the results. From the theory, we can deduce that each quasispecies is the steady-state equilibrium of a separate variation-selection process. This is the same as saying that each quasispecies represents an independent axis of the matrix. Along the axes of the matrix, information is exactly as well as approximately reproduced at an overall distinct rate. You can multiply the eigenvector by the transition matrix, but you really get the same result when multiplying it by its eigenvalue, which is a constant. This means that the quasispecies are self-perpetuating. This property leads to polarization.

Furthermore, we also know that variation more frequently creates similar variants. Therefore, the axes bring together variants that are similar to each other in meaning. Remember that in Test A we built the matrix based on similarity? The theory is the same; only the method to estimate reproduction rates is different. Following the theory, the new matrix must also reflect similarity.

It follows that after all of these procedures and operations, the eigenvectors could be used to predict similarity (or synonymy in the term-document example) better than the initial co-occurrence matrix. This prediction is easy to test, but it really has been tested many times. One could even say that it stood the test of time.

Around the turn of the millennium, the psychologist Thomas Landauer and the computer scientist Susan Dumais have stumbled over exactly this procedure and result. They did not know why they obtained the result and admitted that the technique had a "magical appearance" (Landauer and Dumais, 1997). The circumstances of their discovery are recounted in Context A. Many followers were inspired by this empirical result, but the theoretical interpretation remained a matter of debate.

In comparison to previous work, the new theory derives the mathematical procedure together with the prediction directly from variation-selection processes. Thus, the empirical result is placed in a broader scientific context. The advantage of the theory is that it makes additional interpretations possible that lead to new applications and discoveries.

One prediction is that the eigenvectors can be used to predict not only similarity but also complete opposites. This conclusion can be drawn because variation can lead in many directions. Opposites can easily be reached by negation. 
Another prediction is that every step that brings the co-occurrence matrix closer to the real transition matrix will improve the eigenvectors. This prediction is not tested here, but it might help future technicians develop better algorithms.

A little more interpretation leads to additional predictions. As already mentioned, the eigenvectors obtained through eigendecomposition are the independent axes of the transition matrix. In our case, we simulate dissemination. Let us therefore interpret these axes as dissemination axes.

If the dissemination axes emerge from variation-selection processes, then these processes should be anchored in geographical space and historical time. This would very much reflect the proposition that new varieties emerge coincident in space and time with pre-existent antitypes.

Furthermore, institutions such as funding agencies and publishers should generate their own variation-selection processes. The reasons are easy to illustrate. Funding agencies and publishers have each their institutional scopes and editorial policies. Such scopes and policies support the reproduction of certain types of information at the cost of others. For example, editors select certain contributions while rejecting others. On a large scale, such selective support could become visible as a variation-selection process.

Taken together, we can predict that dissemination axes have institutional, geographical, and historical profiles. Figure 2 is based on a corpus that has been enriched with institutional, geographical, and historical information (methods given in annex). The test confirms that dissemination axes have institutional, historical, and geographical profiles (Fig.2).

The new theory and results allow for one further postulate. As before, dissemination axes are independent from each other. They are realms of speciation. However, we just found that multiple axes, or, to use the initial terminology, multiple quasispecies co-exist. This also means that some other processes must regulate and hold in balance quasispecies evolution. Such processes would have to act against the fastest spreading thought. This postulate is further developed and tested in sections 4-9. 
Fig.2.1 Science and humanities dissemination axes: geographical profiles. Cultural life renews itself unevenly, resulting in regional varieties.

US news, three corpora, thousand dissemination axes, interactive visual online.
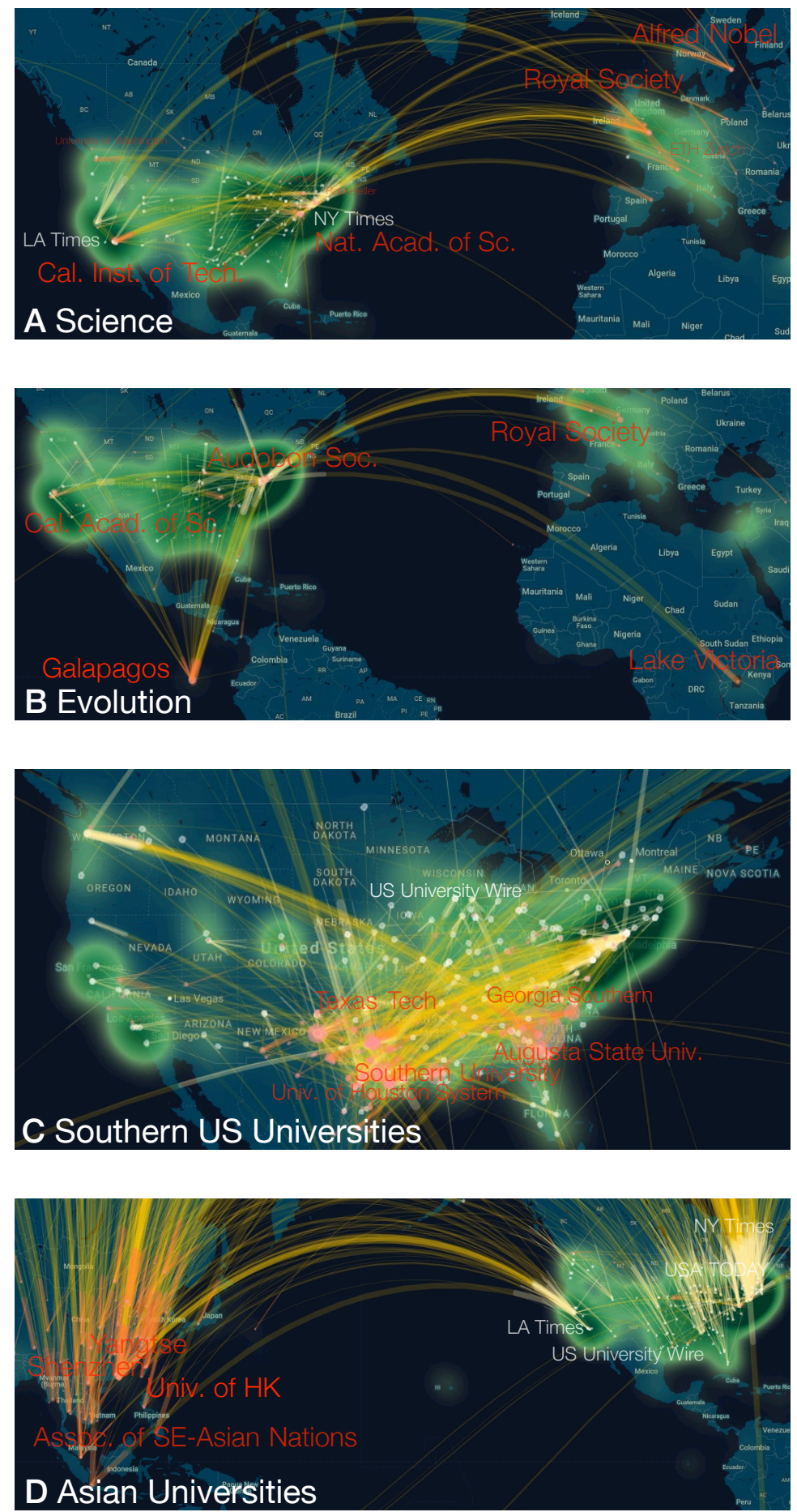

A The largest science dissemination axis in our corpora is themed on recognition and dominated by major research institutions along with the National Academy of Science and the Nobel prize that bridge interest in the various scientific disciplines. (S1.11) B The Galapagos, Lake Victoria, their birds, the California Academy of Science, and the Audubon and Royal societies dominate the discourse on evolution in public media giving its distinct geographical profile. (\$1.129) C Regionally focused, but polycentric, this network unites many institutions in fixed geographical space. $(\$ 1,80)$ D China and south-east asia have many distinct dissemination axes in our corpora, among which this may just be the largest. It is focused on universities and history. (S1,42)

White: publishers

Pink: news content

Lines connect publishers to content they published.

Green: dense zones in the overall corpus distributions. 
Fig.2.2 Science and humanities dissemination axes: institional and historical profiles. With their mission statements and the like, institutions such as publishers and funding agencies create their own variation-selection processes. Variation-selection may also emerge in the realm of collective memory.

US news, three corpora, thousand dissemination axes, interactive visual online.

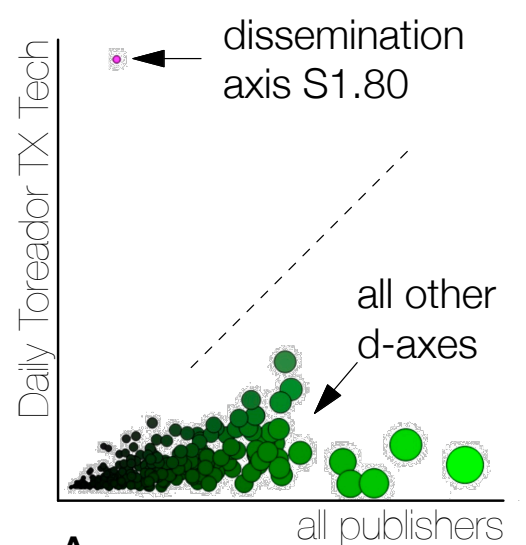

A

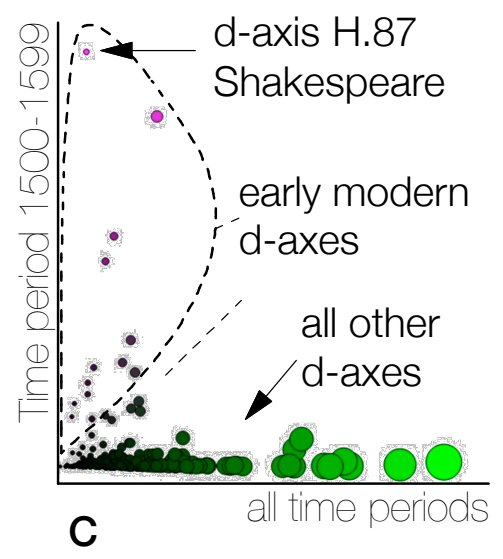

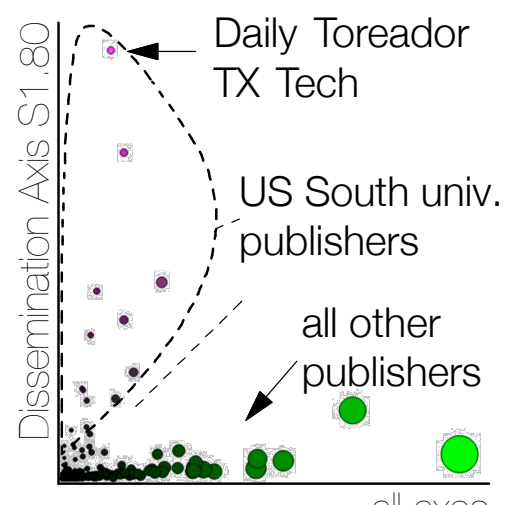

B

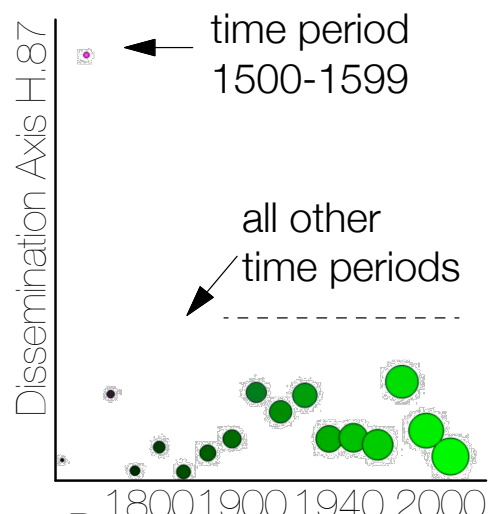

D
A,B Southern US universities (Same dissemination axis as in Fig.1C) This geographically bound network of publishers creates its own dissemination axis that they specialize in (A), and that is uncommon among other publishers (B).

\section{C,D 16th century} dissemination axes. These dissemination axes taken from our humanities corpus root the public discourse in the early modern epoch. The most specialized among them is the one on Shakespeare and early modern drama (A). Other time periods are almost absent from the Shakespeare axis giving it a distinct historical profile (B). 


\section{Context A: Power laws and clusters long lacked explanation.}

Power laws and clustering patterns are distributional laws known for a long time, but they lacked theoretical explanation. Often, the same empirical law motivated different philosophies and politics.

Power laws were discovered at the end of the 19th century (Pareto, 1896). Vilfredo Pareto collected income data and found distributions that looked like pyramids; but those pyramids that he found were so skewed that he also flipped them upsidedown and compared them to spinning tops. Pointed tips supported bulky rotating disks. For Pareto, this distribution illustrated, if not legitimized, the divide between aristocrats and mob. The aristocracy counted few individuals, the mob many.

Felix Auerbach found the same distribution in the census of cities (Auerbach, 1913). In any country, few large cities are surrounded my many small towns, villages, and hamlets. The population counts decrease following a Pareto distribution. A nightly hot-air balloon flight inspired Auerbach to quantify the distribution, but he chose to represent his results differently. After sorting the population counts in decreasing order, he chose to multiply each of these numbers with the corresponding enumeration rank: the largest population count was multiplied by one, the second largest by two, the third by three, etc. The result is a constant value.

In Auerbach's representation, the Pareto distribution has something rather democratic. Ironically, the empirical law is the same. Only the graphical representation and the political interpretation are diametrically opposite.

Alfred Lotka used one of Auerbach's books on physics and counted how many times each author was cited in the book (Lotka, 1926). He also found the same distribution, and his work led to the establishment of bibliometrics as a new field of study. Finally, power laws in natural language became known as Zipf laws when George Zipf popularized them at Harvard (Zipf, 1936).

Many later authors also attested the existence of power laws. For example, they are also known to govern memory. However, the statistical procedure employed in most of the work on power laws is incomplete. The problem is that the data is sorted by the variable under consideration. For example, if you consider word frequency, you should not also sort the data by that same variable (Piantadosi, 2014).

The problem of statistical incompleteness remained unknown for a long time and became a trap for theoreticians. They envisioned processes that produced similar curves. However, the representation chosen by Pareto is, once again, statistically incomplete. Furthermore, the number of curves that one can graphically distinguish is very small. Similar curves may result from different processes, while same processes can be represented with different curves.

If researchers had chosen Auerbach's representation of power laws, they might have realized that more clues are needed to guide the development of good theory. Predicting constant values does not even require a random model. The problem is that, if only constant values need to be predicted, many theories could in principle apply.

Two researchers started fighting with each other when they found out that their proposed processes were not compatible with each other (Simon, 1961). None of their explanations reached consensus. Meanwhile, a second distributional law was discovered that falsifies all past theories (Piantadosi, 2014).

Pareto and Zipf drew straight falling lines. Auerbach discovered constants. However, behind these seemingly neat representations, there are ever-evolving cultural 
groups, circles, partisanship, or hotspots of public attention-behind the straight lines, there are clustering patterns and polarization.

French social scientists may have been ahead of everyone else when they used a class of sophisticated mathematical techniques, dimensionality reductions, to reveal those fateful clustering patterns within the data. Pierre Giraud called these clusters semes. He believed that all culture was composed of 32 semes, and he suggested that power laws could somehow be derived from them (Giraud, 1968). Pierre Bourdieu spoke of fields, imposing a very different interpretation (Bourdieu, 1972). Most notably, he introduced the concept of cultural reproduction.

Later the term meme was coined in a similar context, while dimensionality reductions awakened scientific interest in what became four distinct fields of study: digital sociology and humanities, and computational psychology and linguistics.

The terms digital and computational somewhat reflect that the physical meaning of those computer-driven techniques frequently remained a matter of speculation. Eventually, dimensionality reductions led to the development of the latent semantic analysis. It may seem no happenstance that latent means hidden. Thomas Landauer and Susan Dumais, who developed the algorithm, wrote that its performance seemed magic (Landauer and Dumais, 1997).

Even in absence of theory, dimensionality reductions stood the test of time. The breakthrough of Landauer and Dumais was that they convinced the research community that their results were empirically meaningful. They used their models to predict synonymy; and an entire generation of researchers followed who predicted synonymy with increasing accuracy (Matveeva, Levow, Farahat, and Royer, 2005; Rapp, 2003; Han, 2014; Bullinaria and Levy, 2012).

This empirical path pointed towards a procedure that involves analyzing how multiple thought variants are distributed over separate instances in space and time. Four steps must be performed: First, multiple text documents are collected. Second, the occurrence of distinct terms is counted in each of the documents, and the counts are stored as a document-term matrix. Third, this matrix is manipulated using a procedure called tf-idf. (Procedure mentioned in Test B.) Finally, singular value decomposition is applied to the matrix.

In an alternative setup, the rectangular document-term matrices are transformed into square term-term matrices, after which eigendecomposition is applied instead of singular value decomposition (Matveeva and Farahart). Some 250 topics, as they were now called, seemed a good number of dimensions to reduce the data to.

Mathematically, the algorithm falls into a narrow category of techniques. It took researchers years to introduce probabilistic refinements (Blei, 2003). In parallel, other researchers experimented with splitting documents into sentences and phrases. Separate instances in space and time were defined at increasing fine-grain. Still other researchers replaced the tf-idf procedure with more sophisticated methods.

Despite of all of these empirical developments, the interpretation of what the algorithm is meant to solve was debated and no consensus was reached.

Variation-selection processes resolve the dilemma. They explain clustering patterns based on the principle of variation. Technically, the algorithm proposed by Landauer and Dumais and followed by so many researchers is equivalent to what we discussed in Test B. This means that semes, memes, fields, and topics are dissemination axes. They not only exist but also self-perpetuate as a result of variation-selection processes. 
In addition, the study of variation-selection processes allows us to make further predictions. In Test $\mathrm{B}$, we predicted that the eigenvectors can also be used to predict antonyms; we predicted that the transition matrix can be improved by replacing tf-idf with more sophisticated methods; and we saw that dissemination axes are anchored in geographical space and historical time and may have institutional, historical, and geographical profiles.

Furthermore, variation-selection processes resolve the statistical incompleteness of power laws. Technically, the research concept is to proceed in ways that avoid the sorting procedure initially chosen by Pareto. The data are sorted, but they are sorted using systematics. The sets of intermediary results that are compared to each other are obtained independently, which make the final results statistically complete.

Finally, the presence of multiple parallel variation-selection processes makes us predict that there are other self-regulation processes at work as will be discussed in the following sections.

\section{Variation is often fine-tuned to support flat distribution curves.}

The distributions predicted by variation-selection processes depend on the rates at which information is being reproduced exactly as well as approximately. Similarly, the contours of hilly landscapes depend on how fast the material is elevated, and how fast it rolls to the sides. The relationship between the two processes delimits the possible shapes of topographic formations.

Mathematically, the relationship between exact and approximate reproduction can lead to three main cases (Nowak, 2006a, 39):

The first case represents extreme conditions. Only exact reproduction is present. Consequently, only one variant survives, namely the one that is reproduced fastest. This variant reaches fixation while all others are driven into extinction.

The second case is an intermediate case. Exact and approximate reproduction work in tandem. Selective advantages lead to the formation of hills, but variation flattens them out. The slopes reflect the relationship between variation and selection.

The third case represents the other end of the spectrum. Exact reproduction still exists, but it is completely outweighed by approximate reproduction. Selective advantages then become irrelevant; and the previously hilly landscape becomes fluid.

Variation-selection processes are often found to be at work close to the latter extreme case. Researchers who made this observation have coined the terms "chaos threshold" and "self-organized criticality." These terms reflect that, in many biological systems, only little disturbance can push variation-selection processes beyond a certain threshold at which sensitivity for selective advantages is lost (Nowak, 2000, 89).

It would seem, then, that variation is often fine-tuned to support flat distributions, which allow many different variants to co-exist. Such fine-tuning may have clear evolutionary advantages. Flat distributions are synonymous with broad evolutionary search processes because each additional, surviving variant can potentially guide evolution into new directions.

Nevertheless, there are physical limitations to what is achievable through variation-selection processes alone. The mathematics is again straightforward.

As already mentioned in Test A, variation-selection processes can be simulated through the quasispecies equation, which can be solved using power iteration. 
Distributions and dynamics are simulated by iterative vector-matrix-multiplication: The vector symbolizes the distribution observable in the system; the matrix symbolizes exact and approximate reproduction; and each multiplication symbolizes the passage of time. After repeated multiplications, a so-called dominant eigenvector is obtained. This vector represents the fastest-reproduced, dominant quasispecies.

This means that variation-selection processes broaden evolutionary search from competing variants to competing quasispecies that can guide evolution into multiple directions. However, one single quasispecies eventually comes to dominate, effectively leading evolution into only one direction. This tendency to choose only one final direction is a limiting factor. There is always a road and the road not taken.

The space of possibilities that evolution inhabits can only be much broader than the one choice eventually made by the dominant quasispecies. This observation brings us back to Test $B$ and the question whether there are ways to support multiple quasispecies in parallel. Such co-existing quasispecies would fork evolution into multiple search directions that could be pursued all at the same time. Test B suggested that this latter case is commonly found in culture.

The mathematics gives additional clues. To obtain the next, less fast replicating quasispecies through power iteration, one must first subtract the dominant eigenvector from the matrix. To achieve this same effect in real life, evolution would have to effectively reduce the reproduction rates of the most prolific variants.

Thus, we are led to postulate the existence of some type of regulation that keeps in balance multiple quasispecies by checking the growth of the most prolific variants. Such a process would lead to further diversification.

If the benefits of diversification are larger than the cost of operating the regulation process, such a process can evolve naturally. Let us refer to this idea as diversity selection. It may constitute a different type of cooperation next to direct and indirect reciprocity, as well as spatial, group and kin selection (Nowak, 2006b).

\section{Homeostatic regulation can support additional diversification.}

As already observed above, variation broadens evolutionary search processes. The selection unit is no longer the individual variant, but the quasispecies of variants. Nevertheless, there are limitations to what can be achieved through variation-selection processes alone.

Our postulate is that the benefits of diversification can lead to the evolution of some type of regulation that evens out competition between multiple quasispecies. To achieve this goal, the growth rates of the most prolific variants would have to be checked. Such regulation is typically called homeostasis. Through homeostatic regulation, complex systems maintain optimal working conditions while constantly checking runaway growth and other disadvantageous change.

The first observation that can be made in cultural life in support of this thesis is that omnipresent information looses its value. People become deaf to repeated messages. Information that is reproduced too fast becomes uninteresting. This process is called habituation. Yet, what exactly is habituation; and does it have the desired effects?

Habituation is a homeostatic process found in almost all complex organisms. Humans habituate to omnipresent information; and so do mice. Thomas Insel studied 
voles and showed that a minute mutation in the system that controls habituation leads to crucial adaptations within the animal habitat (Insel and Shapiro, 1992).

Insel's research beautifully illustrates how life renews itself, and how this process is shaped by habituation. Predators threaten prairie voles more than their montane relative. In response, prairie voles evolved to slower habituate to their mates and more rarely leave their homes for mating purposes. In consequence, less habituation also means less diversely recombined genes.

The case of voles supports our concept of diversity selection. The benefits of genetic diversity are the same for all voles. However, homeostatic regulation evolves more strongly in the species in which it is less costly to operate.

The neurochemistry that Insel studied is easily administered through the air, which might be the reason why it was later used in countless studies on human behavior and social judgments (Regan et al., 2016). Let us now study whether habituation can lead to diversification in human culture.

Habituation does not apply to all incoming stimuli whatsoever. Habituation helps the brain manage incoming sensory information by which it is flooded. Towards this purpose, the brain learns to distinguish different stimuli. This process is called discrimination. To keep track of this phenomenon, let us define two thought variants as diverse when habituation discriminates the two and is activated against each of them separately. You do not habituate to life in general, you can discriminate.

Next to discrimination, sensitization constitutes an additional process that modulates habituation. When organisms are sensitized to certain stimuli, previously established habituation is disrupted. Sensitization allows the nervous system to adjust and correct habituation.

One particular type of sensitization is of immediate interest for the study of cultural life. New thought can renew interest in pre-existent thought. Even more broadly, diverse variants can renew interest in any variant at large. Let us call this particular process cross-sensitization. Cross-sensitization controls the interplay between diverse variants of thought.

The next step is to model the time-dependent effects of habituation, discrimination, and cross-sensitization as differential equations. In absence of another name, let us call these equations reception equations. (They simulate how information is received.) As with the quasispecies equation, an equivalent formulation was previously studied in the life sciences. In our case, the assumptions are as follows:

First, habituation is activated at certain rates of efficacy in response to repeated stimuli. Technically, each repeated encounter with a given idea or category of thought activates habituation.

Second, habituation diminishes the spread of ideas or categories of thought at certain rates of efficacy. This assumption captures the phenomenon that people become indifferent to messages that are frequently repeated.

Third, diverse thought may sensitize the audiences. Each encounter with diverse variants may diminish habituation against all variants at certain rates of efficacy. People can regain interest in ideas that seem promising based on new insights.

This setup logically translates into the previously mentioned reception equations (Eqn. 1). The equations take two types of habituation into account. Variant-specific habituation inhibits the spread of specific variants. Cross-reactive habituation inhibits the spread of larger categories of thought. 
Without loss of generality, the reception equations can be further simplified for the study of one diverse variant in isolation. The equations then predict ups and downs of fashions that can be empirically confirmed (Fig. 3 A-B. Test C).

From the test, it can be concluded that habituation effectively reduces the reproduction rates of those prolific variants against which it is activated. In the meantime, variation may already have created variants that escape habituation. Where at first there was one dominant culture, now there is much more thought diversity Cultural life is chased away from dominant culture towards other diverse alternatives and subcultures.

This process achieves the same goal as the matrix operation mentioned in the previous section, in which the dominant eigenvector is subtracted from the transition matrix to make it possible to calculate more eigenvectors. Through homeostatic regulation, multiple quasispecies come to co-exist.

\section{Test C. Habituation cycles.}

The reception equations can be simplified for the study of only one variant. This simplification leaves us with a pair of Lotka-Volterra equations, familiar to most scientists. The equations were named after Lotka (Lotka, 1910), the pioneer of bibliometrics mentioned before, and the mathematician Vito Volterra who popularized them in what became known as mathematical ecology. The equations have since become a textbook example, often used to predict the wavelike wobbling in the sizes of prey and predator populations that consecutively outbalance each other (Nowak, 2000).

Studying habituation against one variant alone requires finding a population that is rather isolated. Writers and audiences would have to fall into one cohesive group of people. The Chicago school of architecture could be a good example. Most Chicago schools are found in the social sciences, while architecture stands apart (Baciu, 2019). Our prediction is that media activity comes and goes in waves.

Given that we already collected everything called Chicago school, all we need is to filter out everything that is not architecture as well as everything that people might habituate against separately.

A closer look into the data reveals that there is the Chicago school of architecture, which is a loose group of architectural practices. However, there also existed registered institutions such as the Chicago School of Architecture Foundation, which was established to support saving the buildings erected by Chicago school architects. Given the clear differences between institution and school of thought, we expect that the nervous system can discriminate between the two. Let us focus on references to the Chicago school of architecture in the narrower sense. (In the worst case, we loose accuracy but gain precision. The latter being what matters most.)

In addition to the problem of disambiguation, we also encounter the problem of redundancy. For example, we might want to include phrases such as the Chicago school of architects that are used almost synonymously with the Chicago school of architecture. There are only few such choices to make. To achieve high statistical precision, the choices have been guided by historical evaluation.

Once problems of disambiguation and redundancy are resolved, we can plot the data over time. Following the Lotka-Volterra model, we expect to observe damped oscillations. 
Between 1960 and 2000, five fashion waves become visible (Fig. 3 B). The second, fourth, and fifth of these waves coincided each with a museum exhibition. The first exhibition was organized by Stephen Prokopoff at the Museum of Contemporary Art; while John Zukowsky at the Art Institute of Chicago curated the latter two. Each of these exhibitions rides the fashion waves. Public interest comes first, tightly followed by the exhibition, and followed by lack of interest.

These observations substantiate that media activity and audience habituation interact with each other in returning waves of fashion. The waves are damped and can be surprisingly regular in shape.

An informal meeting with John Zukowsky in Chicago resolves the question whether exhibition organizers sensed the waves and took advantage of them. Zukowsky says that, as a curator, he was aware of five-year cycles. He judged them important for the museum productions.

At this point, the model does not yet explain why the public attention in the Chicago school of architecture rises so suddenly in the early 1960s, and why it falls in the 21st century. This explanation requires a broader look at all Chicago schools and the interactions that cross-sensitization establishes between them.

Eqn.1 Reception Equations. Homeostatic regulation divided into habituation, discrimination, and sensitization, and mathematically modeled.

$$
\begin{array}{ll}
\frac{d x_{i}}{d t}=x_{i}\left(r-p y_{i}-q z\right), & i=1, \ldots, n, \\
\frac{d y_{i}}{d t}=c x_{i}-b y_{i}-u x y_{i}, & i=1, \ldots, n, \\
\frac{d z}{d t}=k x-b z-u x z . &
\end{array}
$$

The first equation describes the expected count of copies for each diverse variant $x_{i}$ in a larger category $x$. At the next time step, this value increases with $r$, the rate at which the variant is reproduced, but it decreases with habituation as given by the second and third equations. $p$ specifies the efficacy of variant-specific and $q$ the efficacy of crossreactive habituation.

The second equation describes the expected variant-specific habituation $y_{i}$ to diverse variant $x_{i}$. This habituation increases with $c$, the rate at which habituation is evoked, decreases with $b$, the rate at which it is forgotten, and it also decreases with $u$, the ability of the entire category $x$ to sensitize the audience. In a somewhat more complex formulation, the value $x$ can be replaced with $s$, taken to symbolize the diversity index for diverse variants in the broader category $x$.

The third equation describes the expected cross-reactive habituation $z$ to the entire category $x$. This habituation is similar to the previous, but it does not discriminate between variants. Equations adapted from Nowak and May, 2000, 130. 
Fig.3 Large-scale reception. Three homeostatic processes (habituation, discrimination, and sensitization) explain waves of fashion, periods of formation, diversity thresholds, and the transition from history dominated great people to more nuanced cultural diversity and collective rise to fame.

A Media activity and habituation lead to waves of fashion as modeled with Lotka-Volterra equations. B Waves of fashion found in the Chicago school of architecture. Museum exhibitions ride the waves. (Chicago school corpus, 105K perodicals and books, $y=100$ references) C Habituation, discrimination and sensitization lead to a double-phased evolution with a diversity threshold between the two phases, individual spikes on the left and collective rise on the right. During fromation, ideas do not lie dormant, but diversity. This is a textbook case of evolutionary dynamics known to occur in virology. D The phenomena are also found in empirical data, for example in the Chicago schoo/at large. The peak on the left is dominated by one school. The rise on the right is a consequence of collective breakthrough. (Data: google Books, $y=1 / 10 \mathrm{M}$ words)

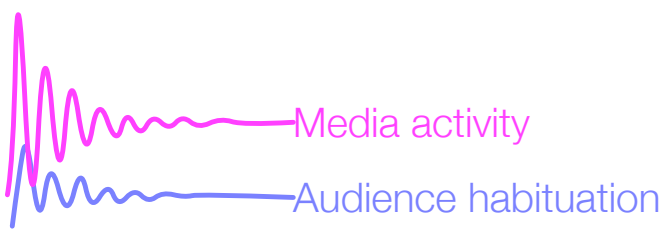

A Habituation cycles
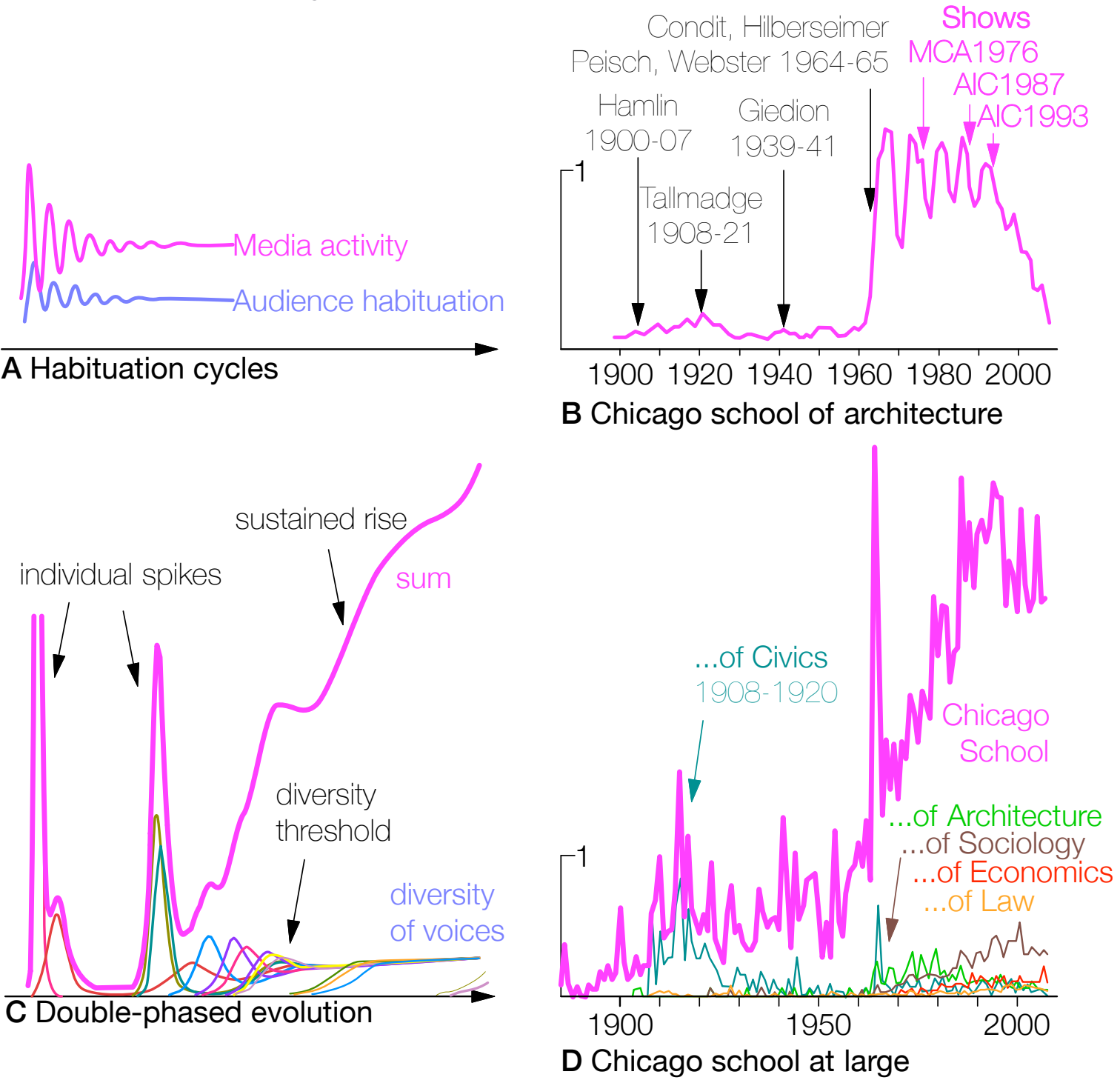


\section{New ideas diversify before coming to breakthrough.}

The previous section has demonstrated that homeostatic regulation can act on variation-selection processes to support additional diversification. However, can this diversification continue forever? The reception equations suggest that there exists a certain diversity threshold. At threshold, habituation is outweighed by crosssensitization. As a result, homeostatic regulation is disrupted, and the reception equations return to quasispecies behavior. However, quasispecies evolution supports less diversification. Thus, initial diversification stops when diversity threshold is reached.

Empirical evidence in support of this prediction is at hand. An initial period of formation is observed in many trends and in the adoption of many technologies (Michel et al., 2011; Aiden and Michel, 2013). The question is how to interpret such periods of formation. The reception equations suggest that ideas do not lie dormant but diversify during formation.

Following this interpretation, initial formative periods are periods of rapid turnover. New ideas may enter a local cultural canon from somewhere else, and new technologies may be unknown to the public until a first application is developed that people can use. These ideas and technological applications may initially exist in only one narrowly definable variant. Once introduced, this variant diversifies. New and newer variants follow on each other's heels. Diversity accumulates. Breakthrough occurs when diversity threshold is reached.

Let us return to our previous example to illustrate and test this prediction. Imagine looking at all Chicago schools next to each other, no matter in which discipline they are. Historical evaluation reveals a century-long period of formation and also confirms our interpretation that Chicago schools did not lie dormant during this period (Baciu, 2018, 2019). New schools were formed across many fields of study. Some of these new schools created spikes of public interest. Eventually, the diversity of schools became overwhelming. People encountered new schools all the time. In the social sciences, these new schools raised interest in all other, similar schools. At this point, diversity threshold was reached and multiple Chicago schools achieved joint breakthrough.

This pattern can also be quantitatively studied and leads to the same conclusions (Fig.3 C-D, Test D). The frequencies of occurrence of Chicago schools in public media can be counted and drawn as a set of line plots. This representation can then be compared to the textbook model of evolutionary dynamics in which the reception equations were initially formulated. One drawing represents a set of simulations, the other a set of line plots gained from analysis of empirical data, but the two drawings look the same. It is not even necessary to change any of the parameters in the simulation despite the fact that this simulation was initially developed for the study of viral evolution rather than cultural life.

Cultural life and organic life are two independent cases. Why then are the parameters of homeostatic regulation so similar? The need for diversification may be the initial force that dictates the magnitudes of the parameters in both cases. These parameters are chosen because they work best at facilitating the evolution of greater diversity. This result further corroborates that diversity selection guides homeostatic regulation. 


\section{Test D. Formative periods and collective breakthrough.}

Historically, the term Chicago school went through a century-long period of formation (Baciu, 2018, 2019). First usage of the term is attested back to 1850, when Chicago had around 30,000 inhabitants. The small town had gone trough dreadful epidemics that left behind a desire for improvements. In response, educational programs were founded in medicine, but East Coast academics did not always approve of the developments. In their judgment, the academic standards in the Midwest were too loose.

Chicagoans knew better, for them, the Chicago school, as they named their emerging school of thought, adapted academic standards to social needs. Today, the Chicago school continues as midway between theory and practice. Nevertheless, the Chicago school in the 19th century was in certain senses unlike the Chicago schools encountered today. A century ago, Chicago schools came and went like fashions. There was no sustained public interest.

In architecture, the idea of a Chicago school is attested since the 1880s and 1890s. In 1893, an educational program was founded, the Chicago School of Architecture. The program united two universities, one offered art, the other technology. Together, they pioneered a midway between art and engineering. Finally between 1900 and 1907, a famous textbook mentioned the Chicago school as a counterpart to the Eastern school. The untrammeled freedom of art in the Midwest had inspired skyscrapers with straight vertical lines that rose from the street uninterrupted to the roofs. Such designs were artistic and practical at the same time. Here again, the Chicago school united theory and practice.

Furthermore, in 1903 and 1904, Chicago schools are encountered in literary fiction and in philosophy, where they were again syntheses of theory and practice (and of reality and romance). The characters of Chicago's writers were fine and frank and spoke truth in fountains of slang. The Chicago school of fiction is closely associated with later schools of art and entertainment, and the Chicago school of philosophy later branched into the Chicago schools of social science.

When plotting the data, two distinct phases become visible in the history of the Chicago school at large. There is a formative period that stretches into the 1950s, followed by a period of growth beginning in the $1960 \mathrm{~s}$. The latter period is marked by collective breakthrough and constant rise in public interest.

During the formative period (1850-1950), the first big spike in public interest in the Chicago school is attested between 1908 and 1921. One single institution, the Chicago School of Civics and Philanthropy, almost wholly dominates the public attention. University of Chicago founded the Chicago School of Civics to unite social theory with social practice. Later, the institution was renamed, but the Chicago school of sociology took over a similar role since the 1920 s.

Finally, in the 1960s, Chicago schools achieved their joint breakthrough. Multiple schools suddenly rose to fame together. The Chicago school of architecture has its fashion waves, while the schools of sociology grow like a quasispecies. They grow apart with sociology eventually dominating over economics and law. During this period (1960-2000), the Chicago school at large grows constantly.

A closer historical evaluation also reveals that Chicago schools may have been most diverse in the 1960s. The number of schools that authors encountered during that period of time overwhelmed them. In the social sciences, this effect awakened new interest across the fields. Authors described a rich, colorful multifold of Chicago schools. 
Since the 1960s, however, diversity has decayed. This pattern is in accord to our prediction that quasispecies behavior returns after diversity threshold is reached. As a consequence, diversification stops and the fastest-growing quasispecies takes over. In our case, the Chicago school of sociology has come to dominate.

\section{Cycles of growth and recession are closely tied to diversification.}

The previous section established that formative periods may not go on forever. Successful diversification is followed by collective breakthrough. Suddenly, groups of ideas rise to fame as a result of successful diversification. Then again, can such success go on forever? After diversity threshold is crossed, homeostatic regulation is diminished. However, that means that diversity can no longer be sustained to the same extent as before. Diversity, which was the initial cause of success, is lost. Consequently, growth must stop.

After explaining fashions as habituation cycles in Test D, we are now led to postulate longer cycles of growth and recession. Let us call them diversification cycles. This name reflects that the process is supported by diversity selection.

Diversification cycles work almost analogous to the mathematician who performs power iteration and transforms the transition matrix in alternating steps in order to obtain multiple eigenvectors. In one step, reproduction leads to runaway growth. In the other, the reproduction rates in the transition matrix are adjusted. Similarly, diversity cycles have two phases: growth and reform. Variation-selection processes shape the earlier phase, homeostatic regulation the latter. The dynamics can be summarized as follows:

Phases of growth set in when diversity threshold is reached and crosssensitization outweighs habituation. As a consequence, variation-selection processes and quasispecies behavior determine the dynamics of growth. A small number of variants reproduce fastest and come to predominate. However, diversity decays over the course of this process. Eventually, habituation is re-activated.

Re-activation of habituation leads to phases of reform. Habituation curbs the growth of the most prolific variants. Reproduction rates are renegotiated. As a consequence, diversity accumulates and eventually rebounds back to threshold conditions.

Let us illustrate the concept of diversitfication cycles with concrete examples and test the predictions. The history of the Chicago school is too new to study repeated cycles of growth and reform. We must choose a different example that has a longer history. How about collecting everything called science? Test B already dealt with a corpus on science and sciences. What would we expect to see over the course of the centuries?

Figuratively, the sciences collectively climb a ladder of success. When all sciences are standing on the same step, they help one of them to climb up, but the one that climbs can only go so far. Homeostatic regulation will eventually convince it to return.

Meanwhile, a new science is ready to climb. In the process, science at large achieves overall growth.

The empirical data supports the predictions. Figure 4 shows clearly distinguishable phases of growth and reform. Growth is fan-shaped. The various science 
branches not only grow-they grow apart in terms of fame. Some branches come to predominate. Diversity decreases.

At this point, reform sets in. The previously growing science branches are curbed as if they were only fashions. Old branches are rediscovered. New sciences rise. Over time, diversity rebounds, and the cycle can restart.

While habituation itself is hard to measure, diversity is more immediately measurable and the threshold levels may remain unchanged. This property allows to also forecast into the future.

Diversification cycles are a surprising type of cooperation that stretches over extended periods of time to reap the benefits of diversity. The homeostatic processes of habituation, discrimination, and sensitization, together with their fine-tuned interplay, may have evolved in cultural life exactly in response to diversity selection.

\section{Test E. Diversification Cycles.}

In section 5, we defined two ideas as diverse when habituation discriminates between the two and is activated against each of them independently. An equivalent definition exists in virology. Two virus strains are defined as diverse if immune response distinguishes between the two strains and acts against each of them independently (Nowak, 2000,148). Some people believe that there is viral news. Here is a definition that blurs the boundary between news and virology. It may be worthwhile noting that the boundary is blurred anyway. The brain is involved in both the creation of culture and the control of certain immune responses (Baciu, 1945; Baciu, 1988).

Following the definition given above, diversity is subjective. The paths taken by cultural diversification depend on the process of discrimination, and therefore on human perception. In response to this observation, the research concept is to rely on linguistic differences that evolve in entire groups of authors and audiences. This procedure puts human perception at the center, while also making detailed expert knowledge unnecessary.

Let us evaluate the evolution of science and diverse science branches as proposed in section 7. We shall focus on phrases that contain the terms science or sciences. This allows for a straightforward system of analysis. Diverse science branches are the natural, physical, social, political and other sciences. The underlying assumption is that people call something physical science when they wish to emphasize that physics is a branch of science, but they nevertheless distinguish this particular science branch from others.

The next step is to compute diversity from counts of diverse science branches. A basic approach could consist in estimating the likelihood that a person in the audience encounters two science branches in a sequence. The higher this likelihood is, the higher the experienced diversity.

In ecology and virology, Simpson's diversity index reflects an equivalent measure, namely the probability that any two species meet. Simpson's diversity index does not tell the total number of species in a habitat. (Ecologists call that "richness.") Instead, it characterizes the shape of the distribution. Typically, the index is given as $1 / \mathrm{D}$. Low values indicate steep distribution curves. High values indicate high diversity.

Let us calculate diversity using Simpson's formula. Each of the diverse science branches is counted as a species. What, then, should the diversity index predict following the reception equations? 
The equations make the connection between diverse variants and larger categories of thought. In our example, all sciences contribute to the larger domain of knowledge people consciously reference as science. The growth of science can be directly measured by counting how many times people reference the term science in their writings. Our hypothesis is that the diversity index can be used to predict the growth and decay of science at large. We expect that diversity index and science growth move in opposite directions. When science does not grow, diversity accumulates until threshold is reached. After that, science grows but diversity decays. Eventually, diversity reaches low levels and science stops growing. At this point, the cycle restarts.

Empirical data confirms the predictions. One particular observation should not be missed. Overall, science grows, but diversity stays between threshold levels. This property may facilitate forecasts. 
Fig.4 Growth and stagnation as diversification cycles. Diversification leads to growth, but growth reduces diversity and comes to a halt. Diversification restarts. These predictions are confirmed by measuring growth and Simpson's diversity index. In both simulation and empirical data, growth and diversity index move against each other. Diversity index stays between thresholds over three centuries of "science."

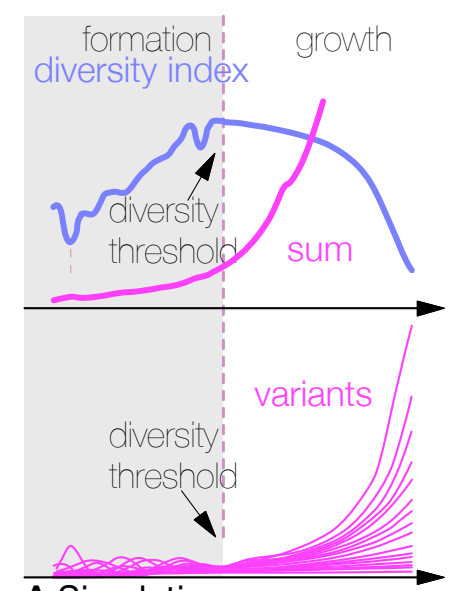

A Simulation.

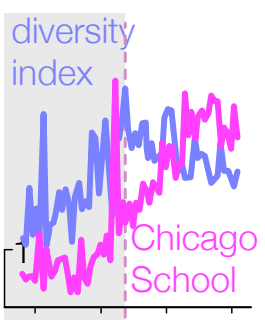

Chicago

5

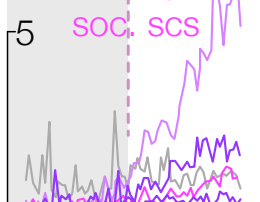

1940 2000 1900

B Chicago, 60 y. C Univ. of Calif., 100 years

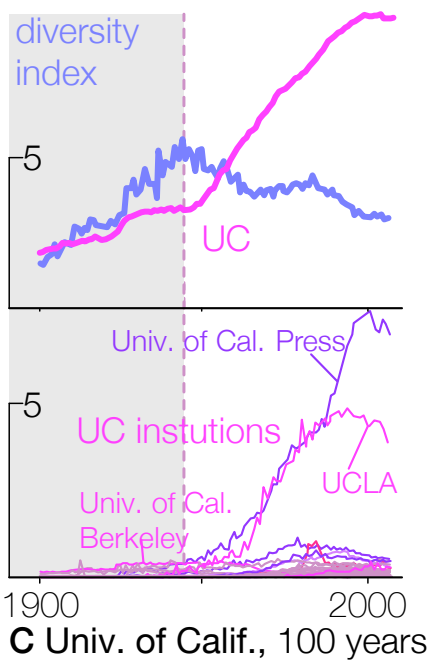

D Science

250 years,

3 cycles.

Science grows.

Diversity index stays between thresholds.

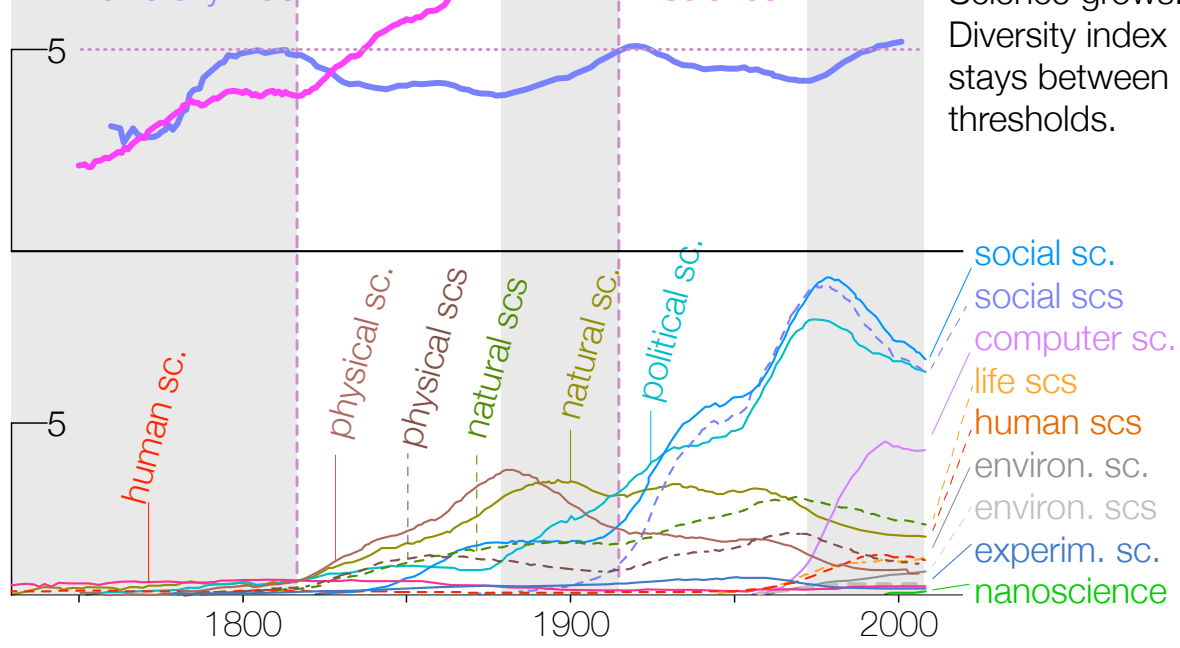

A Diversity and growth delimit each other. Simulation by Nowak et al. 1991, initially developed for a different application. B Diversification in Chicago schools of social science. "Chicago School"/"Chicago" y=1/1 M words, Chicago schools of social science $y=1 / 1 \mathrm{M}$ words, Diversity Index $y=1 / \mathrm{D}$. C Diversification in institutions of the University of California. "University of California"/"California" y=2/100 words; Diversity index y=1/D; institution names $y=1 / 1 \mathrm{M}$ words. D Science and science branches. "Science" $y=2 / 10 \mathrm{~K}$ words; Diversity index y=1/D; Science branches y=1/1M words. Data: google Books. 


\section{Diversification supports the evolution of common ethos.}

The concept of cross-sensitization not only led us to predict formative periods and diversity cycles, but it also implies that entire bodies of knowledge can share certain characteristics. For example, science branches share parts of their name, and so do Chicago schools. In addition, a common ethos can evolve to unite all sciences and many schools of thought. The study of variation-selection processes and homeostatic regulation can be expanded to shed additional light onto this phenomenon.

The starting point is that there is a slight difference between initial formation and later phases of reform. The cultural richness that is initially established during formation is not completely lost during later phases. Even during growth, the total number of variants does not necessarily decrease. Only the distribution becomes less even. Under these circumstances, growth and reform re-arrange dominance but do not need to be as creative as initial formation. As a result, a common ethos evolves.

A closer look at historical data illustrates the process in additional depth. Historic evaluation reveals that most Chicago schools deal with midways of theory and practice. Many such midways have been proposed in Chicago. Researchers are encouraged to study them in the city, and audiences are avid to learn about new results. Midways drive the dissemination of Chicago schools since their initial formation. Let us call this type of attribute dissemination driver.

During growth and reform, dissemination drivers may spread to multiple cultural settings. To take the previous example, the theme of the Chicago midway is not domainspecific but has spread across architecture, urbanism, as well as life and social science. In the process, diversification did not completely re-invent what makes a Chicago midway. After all, cross-sensitization requires cross-recognition. Some shared characteristics are necessary to allow for cross-recognition.

Nevertheless, the spread of Chicago midways to each new cultural setting added a sense of diversity. Once bored of one particular Chicago school, there are plenty of other similar ones to renew your interest. Thus, the cultural settings into which the Chicago school penetrated enhanced public reception in the audiences. Let us classify the cultural settings as diversification facilitators.

A similar distinction between dissemination drivers and diversification facilitators can be found among science branches. The scientific method is a dissemination driver, while the various fields of study are diversification facilitators. As new scientific fields evolve, they not only come to share parts of their name. They also share the scientific method as their common ethos. The general audience rediscovers this theme and learns to appreciate it across multiple fields of study. The disciplines do not change the essence of the scientific method but nevertheless broaden the scope of science at large.

Figuratively, imagine a library. Dissemination drivers are the scope of the library. Books are acquired if they contribute to this scope, in the first place. Nevertheless, libraries do not consists of only one book, copied to fill all shelves. Each shelve may enrich the reader's views with a new perspective. The shelves constitute the library's diversity.

Technically, the distinction into two types of attributes reflects the different workings of variation-selection processes and homeostatic regulation. Dissemination drivers evolve to fuel variation-selection processes. On the other hand, diversity facilitators evolve in response to homeostatic regulation. 
This division of labor also leads to an important conceptual separation between initiators and followers. The cultural setting or fields in which thought is first formed are the most creative and discover most dissemination drivers. In contrast, followers primarily perpetuate a common ethos. The same distinction can be made between trendsetters and followers.

These postulates can be qualitatively tested through historical evaluation (Baciu, $2018,2019)$. The Chicago school of architecture is not only one of the oldest Chicago schools but maybe also the richest in terms of dissemination drivers. In architecture, there are two very distinct types of Chicago school. One type proposed urban midways of art and science and engaged with self-organization and dispersed knowledge. The other is the Prairie school that emerged in the context of architectural licensing and institutional regulation. These two types of Chicago school are opposed, given that one is about urban life and self-organization while the other is closely tied to suburban settings and institutional regulation. The latter school is newer, and it did not spread to other disciplines. Architecture remains richer than other fields because it has these two distinct Chicago schools.

The distinction between dissemination drivers and diversity facilitators, together with the insight that evolutionary processes find most of the dissemination drivers during initial formation, applies beyond cultural life. Similar phenomena are also found in the life sciences. Cancer driver genes are those genes that drive the growth of both tumors and metastases. However, the initial tumors are the place where most of the driver genes evolve. Metastases only spread an already formed disease to new places and organs (Reiter et al. 2018, 2019).

\section{Varieties permeate all life.}

Not only cultural life, but also life in general must constantly renew itself, often without change. The same paths are beaten many times. Consumer goods and consumables are standardized and mass-produced. Useful processes are automated. And species of living organisms die out if they do not reproduce.

Change only accompanies reproduction. Inventions depart from previous knowledge and norms. New varieties evolve as by-product of reproduction. Would this mean that many of our conclusions about cultural life also apply more broadly to technology and life science?

The observation that new varieties emerge coincident in space and time with preexistent antitypes, which accompanied us throughout this article, does not only apply to culture. Alfred Russell Wallace formulated this statement in biology as early as 1857 (Wallace, 1857). His discoveries were similar to the ones we made in cultural life. Eventually, his observations led him to formulate evolution as a process of variation and selection. He wrote that varieties depart further and further away from the original types, and that selection within fixed physical environments is a limiting factor (Wallace, 1858).

Wallace's research in the Amazon and the Malay Archipelago was deeply inspired by geographical boundaries such as large rivers and oceans. Regional varieties similar to the ones we mapped out in cultural life are also found among animal species-they initially inspired Wallace's articles.

Even the term quasispecies, although with a slightly different meaning, is attested back then in the 1860s. Francis P. Pascoe, who sorted out Wallace's and other collections 
of beetles from the Malay Archipelago, classified the varieties brought to him into both species and quasispecies (Pascoe, 1866, 258).

On the Galapagos Islands, Charles Darwin made observations similar to Wallace; and he brought evolutionary thinking to international breakthrough (Darwin, 1859). Meanwhile, island biogeography has transformed ecology from descriptive field into theoretical and empirical science (McArthur and Wilson, 1963, 2001; Patino et al., 2017).

In addition, there are many other ties between cultural life and life science. Fashions, formative periods, diversity thresholds, and the evolution of common ethos are phenomena not restricted to cultural life. Similar quantitative phenomena are also found in inter-species evolution, virus infections, and cancer (Nowak, 2000; Hill, Rosenbloom, Nowak, Siliciano, 2018; Reiter, 2018, 2019). Furthermore, power laws are not only known as Lotka's laws in bibliometrics and as Zipf laws in the humanities. In biology, they are known as Willis laws.

It is all the more fascinating to realize that not only some of the most pervasive real-world phenomena transcend cultural life, but also their theoretical explanations are equivalent in cultural life and life science. The concept of exact and approximate reproduction was an important part of Shannon's theory of communication (Shannon, $1948,1949)$. Later, this concept proved unavoidable in the study of variation-selection processes, in particular towards the formulation of the quasispecies equation (Eigen, 1971). In that context, variation is defined as approximate reproduction, which is our definition, too. Moreover, homeostatic regulation plays an important part not only in cultural life, but also in the life sciences (Baciu, 1980, 1988; Baciu and Arama, 1994; Schulkin, 2003). The reception equations already are a textbook example of evolutionary dynamics. Identical and similar equations were applied to virology and ecology for over a century (Nowak, 2000, 7; Nowak et al., 1991; Nowak, Anderson, May, 1990; Regoes, Wodarz, Nowak, 1998).

The comparison of nature and culture reveals differences, too. Variants connected by random genetic variation are not the same as variants connected genetic recombination in complex organisms, or variants connected by human creativity. The general structure of the processes is the same, but overall efficiency increases dramatically. Variation connects, selection selects, and homeostasis distinguishes between different groups of copies. The matrix that interconnects variants becomes increasingly efficient in facilitating new, meaningful diversification.

However, if variation, selection, and homeostasis are of such broad consequence in nature and culture, should we not expect that researchers have already observed some equivalence between cultural life and life science?

Wallace wrote not only about the varieties of so-called paradise birds, but also about the varieties of names that people and commerce had given to those same birds. The birds evolved, and so did their names. Other researchers do not stand behind. Maybe more consciously than any other, Lotka united the study of multiple disciplines. He formulated a third law of thermodynamics, believing that this law governed nature as well as the evolution of human technology (Lotka, 1945). The essence of his statement is that evolution increases the efficiency of the matrix, as we have seen above.

Lotka's third law of thermodynamics was later rethought and reformulated by many researchers, among them Norbert Wiener and Ilya Prigogine. More recently, Adrian Bejan was awarded the Benjamin Franklin Prize for the constructal law, which further refines and enriches this research direction with theory and examples from across physics, nature, and technological evolution (Bejan, 2016, 2020). Variation- 
selection processes negate entropy and the efficiency of the matrix increases in our case, too.

Expanding on Lotka's work, we can now only propose that the patenting of technological innovation, free markets, and governmental regulation are logical counterparts of variation, selection, and homeostatic regulation. This hypothesis could be supported by many facts: first, taxation is usually set to increase with income category; second, antitrust laws act against large companies; third, groups of companies discover lobbying, which is aimed at sensitizing audiences; fourth, economies are constantly struck by cycles of growth and recession; and fifth, growth dominated by small numbers of industries is not only common but also unsustainable.

Even beyond science and society, the equivalences stated in this article have found expression in daily culture. Loosely speaking, there is viral news and each personal relationship has its own chemistry. There are urban cultures, agriculture, and scientific cultures. There are human bodies and bodies of knowledge. People use the same words for cultural life and life science, and they are right. There exist equivalences between nature and culture.

\section{Reconciling unity and diversity.}

The discovery of variation-selection processes and homeostatic regulation across both cultural life and life science may be no happenstance. Diversity selection may have driven the evolution of these processes in both cases.

Diversification and ensuing specialization can yield many benefits, many of which stem from the fact that physical and natural resources are inconstant. Seasons are changing, continental plates are shifting, and evolution transforms life.

Diversification and specialization may help living systems make efficient use of changing resources at any given time. In addition, diversified systems may be more resilient against partial disruption within the physical environment, and diversity helps evolution search in many directions in parallel. With diversification, evolution can take at once the road and the road not taken. As long as such benefits outweigh the cost for operating the processes that sustain diversification and specialization, diversity selection can persist and prevail.

In our case, variation-selection processes search as broadly as possible, and homeostatic regulation allows for additional diversification. The cost of running these processes is cognitive load as well as variants that are constantly discarded. After all, diversification is a process of trial and error; and error has a cost to it.

On the other hand, diversification may bring many benefits of scientific discovery and technological achievement. As long as variation-selection processes and homeostatic regulation can help faster achieve and better support meaningful diversification, such processes are likely to evolve. The system in its entirety is likely to support diversification.

In principle, diversification can occur in many systems. Among them are physical and biological systems, social organization, and cultural value systems. Diversification can be beneficial in many different contexts. Biological systems evolved to use all kinds of natural resources; and urban life is inseparable from division of labor into many similar, yet specialized tasks. Diversification is everywhere.

Accordingly, the relationship between unity and diversity has long fascinated humanity. The two words are united in something as vast as uni-verse and as 
intellectually fulfilling as uni-versity. In addition, the fascination with diversification is deeply rooted in ancient history. The parable of the Tower of Babel was interpreted in many ways, putting tower and joint achievement next to linguistic diversification and misunderstandings. The relationship between unity and diversity is reflected in ancient history by the two words Babel and babbling.

Edward Simpson, who formulated the diversity index that we utilized, studied the relationship between unity and diversity in a more scientific context. He made a peculiar observation. At different scales, data invites divergent interpretations that cannot be pursued all at the same time. Small clusters may point one way, large ones may point the other. Similar observations were later made in the study of psychology and group dynamics (Baciu, 1982).

Today, the idea of unity leaves us with the World Economic Forum while diversity is alive in social media and postmodernist theories. This present article has attempted to go a step further, holding that diversification no less than fuels collective growth no matter whether this growth is physical, biological, economical, or cultural. Diversity selection is the main force behind the process.

Finally, the newly discovered equivalences between cultural life and life science, as well as the understanding that unity and diversity need each other, could further inspire us to propose that science and humanities should not be strictly separated. Human science was the first of the sciences. From it, physical, natural, social, and other sciences split over the centuries. Maybe it is time that human science is enriched with mathematical precision, which can reunite it with its sister sciences, as in this present article. 


\section{Bibliography}

Abramova, A., \& Baciu, D. C. (2020a). Environmental Awareness Lagging Behind Pollution: Local Impact of Coal Mining in Spitzbergen and the Formation of Public Opinion. Annual Meeting of the American Association of Geographers. Denver, CO.

Abramova, A., \& Baciu, D. C. (2020b). Spitzbergen Arctic Landscapes, Coal Mining Impact, and the Rise of Environmental Awareness. Sixth International Symposium on Arctic Research. Tokyo.

Aiden, E., \& Michel, J. B. (2014). Uncharted: Big data as a lens on human culture. New York, NY: Penguin.

Auerbach, F. (1913). Das Gesetz der Bevölkerungskonzentration. Petermanns Geographische Mitteilungen, Vol. 59, 74-76.

Baciu, D. (1982). Doctoral dissertation, University of Medicine and Pharmacy. Cluj-Napoca.

Baciu, D. C. (2016). Sigfried Giedion: Historiography and History of Reception on a Global Stage. Ar(t) chitecture, 40-52.

Baciu, D. C. (2017). The Chicago School: Evolving systems of value. Technical Report. HathiTrust Research Center.

Baciu, D. C. (2018). From everything called Chicago School to the theory of varieties (Doctoral dissertation, Illinois Institute of Technology).

Baciu, D. C. (2019). Chicago Schools: Large-scale dissemination and reception. Prometheus, Vol. 2, 20-43.

Baciu, D. C. (2020a). Cultural Life: Theory and Empirical Testing. OSF Preprints. DOI: $10.31219 /$ osf.io/ad9fu

Baciu, D. C. (2020b). Sigfried Giedion's Chicago School: A Midpoint in 150 Years of History. OSF Preprints. DOI: 10.31219/osf.io/fh2gj

Baciu, D. C. and Abramova A. (2020), Svalbard's Arctic Settlements: From Mining Sites to Urbanized Environments. EGU. DOI: 10.5194/egusphere-egu2020-2458

Baciu, I. (1980). Homeostazia Oxigenului. Dacia: Cluj-Napoca.

Baciu, I. (1988). Nervous Control of the Phagocytic System. International Journal of Neuroscience, Vol. 41(1-2), 127-141.

Baciu, I., \& Aramă, O. (1994). Integrative cybernetic model of oxygen homeostasis. Romanian Journal of Physiology: Physiological Sciences, 31(1-4), 25-45.

Baciu, I., Hriscu, M., \& Saulea, G. (2003). Hypothalamic mechanisms of immunity. International Journal of Neuroscience, Vol. 113(2), 259-277. DOI: 10.1080/00207450390162065.

Bejan, A. (2016). The physics of life: the evolution of everything. New York, NY: St. Martin's Press.

Bejan, A. (2020). Freedom and Evolution. Basel: Springer.

Benetato, G., Baciu, I., \& Vlad, L. (1945). Zentralnerven-system und Abwehrfunction. Die Rolle der hypothalamischen vegetativen Zentren bei der Phagozytentatigkeit Schweiz Med Wochenschr, Vol. 75, 702-712.

Bernhard, R. M., Chaponis, J., Siburian, R., Gallagher, P., Ransohoff, K., Wikler, D., ... \& Greene, J. D. (2016). Variation in the oxytocin receptor gene (OXTR) is associated with differences in moral judgment. Social Cognitive and Affective Neuroscience, Vol. 11(12), 1872-1881. DOI: $10.1093 / \mathrm{scan} / \mathrm{nsw} 103$

Bertels, F., Gokhale, C. S., \& Traulsen, A. (2017). Discovering complete quasispecies in bacterial genomes. Genetics, Vol. 206(4), 2149-2157. DOI:10.1534/genetics.117.201160

Besnard, A., Caboche, J., \& Laroche, S. (2012). Reconsolidation of memory: a decade of debate. Progress in Neurobiology, Vol. 99(1), 61-80. 
Blei, D. M., Ng, A. Y., \& Jordan, M. I. (2003). Latent dirichlet allocation. Journal of Machine Learning Research, Vol. 3, 993-1022.

Bourdieu, P. (1972). Outline of a Theory of Practice. Cambridge: Cambridge university press.

Bullinaria, J. A., \& Levy, J. P. (2012). Extracting semantic representations from word cooccurrence statistics: stop-lists, stemming, and SVD. Behavior Research Methods, Vol. 44(3), 890-907.

Darwin, C. (1859). On the origin of species by means of natural selection or the preservation of favoured races in the struggle for life. Oxford: Oxford University Press.

Darwin, C., \& Wallace, A. (1858). On the tendency of species to form varieties; and on the perpetuation of varieties and species by natural means of selection. Journal of the Proceedings of the Linnean Society of London. Zoology, Vol. 3(9), 45-62.

Domingo, E., Schuster, P., \& Oldstone, M. B. (Eds.). (2016). Quasispecies: from theory to experimental systems (Vol. 392, p. 357). Switzerland: Springer.

Eigen, M. (1971). Selforganization of matter and the evolution of biological macromolecules. Naturwissenschaften, Vol. 58(10), 465-523.

Giraud, P. (1968). The semic matrices of meaning. Information (International Social Science Council), Vol. 7(2), 131-139.

Han, L. (2014). Thesis. University of Mariland, Baltmore, MD.

Henrich, J. (2017). The secret of our success: How culture is driving human evolution, domesticating our species, and making us smarter. Princeton, NJ: Princeton University Press.

Hill, A. L., Rosenbloom, D. I., Nowak, M. A., \& Siliciano, R. F. (2018). Insight into treatment of HIV infection from viral dynamics models. Immunological Reviews, Vol. 285(1), 9-25. DOI: 10.1111/imr.12698.

Insel, T. R., \& Shapiro, L. E. (1992). Oxytocin receptor distribution reflects social organization in monogamous and polygamous voles. Proceedings of the National Academy of Sciences, Vol. 89(13), 5981-5985.

Landauer, T. K., \& Dumais, S. T. (1997). A solution to Plato's problem: The latent semantic analysis theory of acquisition, induction, and representation of knowledge. Psychological Review, Vol. 104(2), 211-240.

Lotka, A. J. (1910). Contribution to the theory of periodic reactions. The Journal of Physical Chemistry, Vol. 14(3), 271-274.

Lotka, A. J. (1926). The frequency distribution of scientific productivity. Journal of The Washington Academy of Sciences, Vol. 16(12), 317-323.

Lotka, A. J. (1945). The law of evolution as a maximal principle. Human Biology, Vol. 17(3), 167-194.

MacArthur, R. H., \& Wilson, E. O. (1963). An equilibrium theory of insular zoogeography. Evolution, Vol. 17(4), 373-387.

MacArthur, R. H., \& Wilson, E. O. (2001). The theory of island biogeography (Vol. 1). Princeton, NJ: Princeton university press.

Matveeva, I., Levow, G., Farahat, A. \& Royer, C. (2005). Term representation with generalized latent semantic analysis. In N. Nicolov, K. Bontcheva, G. Angelova, \& R. Mitkov (Eds.), Recent advances in natural language processing $I V$ : selected papers from RANLP, (pp. 45-54). Amsterdam: John Benjamins Publishing Company

Matveeva, Irina and Azman Farahat. Generalized Latent Semantic Analysis. Patent US8312021B2.

Michel, J. B., Shen, Y. K., Aiden, A. P., Veres, A., Gray, M. K., Pickett, J. P., \& Pinker, S. (2011). Quantitative analysis of culture using millions of digitized books. Science, Vol. 331(6014), 176-182. 
Nowak, M. A. (1992). What is a quasispecies?. Trends in Ecology \& Evolution, Vol. 7(4), 118-121. DOI:10.1016/0169-5347(92)90145-2.

Nowak, M. A. (2006a). Evolutionary dynamics: exploring the equations of life. Cambridge MA: Belknap Press

Nowak, M. A. (2006b). Five rules for the evolution of cooperation. Science, Vol. 314(5805), 1560-1563.

Nowak, M. A., Anderson, R. M., McLean, A. R., Wolfs, T. F., Goudsmit, J., \& May, R. M. (1991). Antigenic diversity thresholds and the development of AIDS. Science, Vol. 254(5034), 963-969.

Nowak, M. A., May, R. M., \& Anderson, R. M. (1990). The evolutionary dynamics of HIV-1 quasispecies and the development of immunodeficiency disease. Aids, Vol. 4(11), 10951103.

Nowak, M., \& May, R. M. (2000). Virus dynamics: mathematical principles of immunology and virology: mathematical principles of immunology and virology. Oxford: Oxford University Press.

Pareto, V. (1896). Cours d'économie politique, Rouge. Lausanne, Switzerland.

Pascoe, F.P. (1866). On the coleoptera of Penang. Proceedings of the Zoological Society of London, May 8, 222-267.

Patiño, J., Whittaker, R. J., Borges, P. A., Fernández-Palacios, J. M., Ah-Peng, C., Araújo, M. B., ... \& de Nascimento, L. (2017). A roadmap for island biology: 50 fundamental questions after 50 years of The Theory of Island Biogeography. Journal of Biogeography, Vol. 44(5), 963-983.

Piantadosi, S. T. (2014). Zipf's word frequency law in natural language: A critical review and future directions. Psychonomic Bulletin \& Review, Vol. 21(5), 1112-1130. DOI: 10.3758/s13423-014-0585-6

Rapp, R. (2003). Word sense discovery based on sense descriptor dissimilarity. Proceedings of the ninth machine translation summit (pp. 315-322).

Regoes, R. R., Wodarz, D., \& Nowak, M. A. (1998). Virus dynamics: the effect of target cell limitation and immune responses on virus evolution. Journal of Theoretical Biology, Vol. 191(4), 451-462.

Reiter, J. G., Baretti, M., Gerold, J. M., Makohon-Moore, A. P., Daud, A., IacobuzioDonahue, C. A., ... \& Vogelstein, B. (2019). An analysis of genetic heterogeneity in untreated cancers. Nature Reviews Cancer, Vol. 19(11), 639-650.

Reiter, J. G., Makohon-Moore, A. P., Gerold, J. M., Heyde, A., Attiyeh, M. A., Kohutek, Z. A., ... \& Zucker, A. (2018). Minimal functional driver gene heterogeneity among untreated metastases. Science, Vol. 361(6406), 1033-1037.

Schulkin, J. (2003). Rethinking homeostasis: Allostatic regulation in physiology and pathophysiology. Cambridge, MA: MIT Press.

Shannon, C. E. (1948). A mathematical theory of communication. Bell System Technical Journal, Vol. 27(3), 379-423.

Shannon, C., \& Weaver, W. (1998). The Mathematical Theory of Information, 1949. Urbana, IL: University of Illinois Press.

Shaw, J. (2016). The memory illusion: Remembering, forgetting, and the science of false memory. London: Random House.

Simon, H. A. (1961). Reply to Dr. Mandelbrot's post scriptum. Information and Control, Vol. 4(2-3), 305-308.

Sparck Jones, K. (1972). A statistical interpretation of term specificity and its application in retrieval. Journal of Documentation, Vol. 28(1), 11-21.

Wallace, A. R. (1857). XLV.-On the natural history of the Aru Islands. Annals and Magazine of Natural History, Vol. 20(121), 473-485. 
Wallace, A. R. (1858). On the tendency of varieties to depart indefinitely from the original type. Proceedings of the Linnean Society of London, Vol. 3(62), 53-62.

Wallace, A.R. (1969). The Malay Archipelago. London: Macmillan.

Zipf, G. K. (1936). The psycho-biology of language: An introduction to dynamic philology. Abingdon: Routledge.

Note on this preprint: This article has been in peer-review for more than one year since April 2019, which made me decide to make it available as preprint. The article is written for humanities scholars but reflects the theory also discussed for life scientists in my "Cultural Life: Theory and Empirical Testing." 